\title{
Optical/Ultraviolet Continuum Polarization of AGN Accretion Disks
}

\author{
Omer Blaes and Eric Agol \\ Physics Department, University of California, Santa Barbara, CA 93106
}

\begin{abstract}
We show how the effects of Faraday rotation and a detailed treatment of the radiative transfer can address various aspects of the polarization problem of AGN accretion disks. In particular, we show how accretion disks might explain the rises in polarization observed blueward of the Lyman edge in several quasars.
\end{abstract}

\section{Introduction}

Accretion disks currently face a number of severe problems in explaining the optical and ultraviolet light that we observe from active galactic nuclei (AGN) (e.g. Barvainis 1993). Here we wish to concentrate on just one of them - the continuum polarization. Calculations of the polarization of AGN accretion disk radiation are still fairly primitive. Because the opacity in the inner regions of the disk is often thought to be dominated by electron scattering, and a thin disk basically has planar geometry, the polarization has until recently been assumed to be that of a plane-parallel, optically thick, pure electron scattering atmosphere (Chandrasekhar 1960). This implies that the radiation will be 11.7 percent linearly polarized for a disk viewed edge-on, with the polarization decreasing to zero as the disk is viewed more and more face-on. The plane of polarization will be parallel to the disk plane. These predictions are contrary to the observations, where small continuum polarizations are observed which are generally parallel to the radio axes in type 1 objects (e.g. Antonucci 1992).

Laor, Netzer, \& Piran (1990) improved the theory by including the effects of relativity and the frequency-dependent absorption opacity $\kappa_{a b}$. The latter is not in fact negligible compared to the electron scattering opacity $\kappa_{e s}$, and they simply assumed that locally the emerging polarization was given by the Chandrasekhar value $P_{C}$ times a reduction factor, i.e.

$$
P=P_{C} \frac{\kappa_{e s}}{\kappa_{e s}+\kappa_{a b}}
$$

The reduction factor is a function of optical depth in the atmosphere, and they simply used the value at the local disk photosphere for the frequency of interest. This formula is reminiscent of an analytic calculation of the effects of absorption opacity on polarization by Gnedin \& Silant'ev (1978), but it is not the same. The polarization is only proportional to this reduction factor in the limit $\kappa_{a b} \gg \kappa_{e s}$, and even then it is not proportional to $P_{C}$. A consequence of Laor et al.'s (1990) formula is that the predicted polarization is guaranteed to be less than or equal to 
the Chandrasekhar value. In addition, the polarization should drop blueward of the Lyman limit due to the strong increase in hydrogen photoionization opacity.

\section{Faraday Rotation by Magnetic Fields in the Disk Photosphere}

It is becoming increasingly clear that magnetic fields play a central role in the dissipative processes occurring in many types of accretion disk, and AGN are no exception. A number of authors have realized that such fields could destroy the polarization signature of an AGN accretion disk (e.g. Blandford 1990). Equipartition fields in the inner regions of the disk are $\sim 10^{4} \mathrm{G} \alpha^{-1 / 2}\left(M / 10^{8} M_{\odot}\right)^{-1 / 2}$, where $\alpha$ is the viscosity parameter and $M$ is the mass of the black hole. The Faraday rotation angle for radiation at wavelength $\lambda$ is $\theta \simeq 0.1 \tau_{e s} B_{\|}(\lambda / 5000 \AA)^{2}$ radians, where $\tau_{e s}$ is the Thomson depth along the line of sight near the photosphere and $B_{\|}$is the magnetic field in $\mathrm{G}$, also along the line of sight. Equipartition fields are easily strong enough to depolarize the optical radiation field emerging from the inner parts of the disk.

An approximate analytic calculation of the effects of Faraday rotation by a uniform field in a pure scattering atmosphere was performed by Silant'ev (1979), and applied to accretion disks in X-ray binaries by Gnedin \& Silant'ev (1977). We have recently improved upon these treatments by calculating the radiative transfer numerically, generalizing the possible field topologies to include random field orientations, and integrating the resulting polarized spectrum over an AGN accretion disk model (Agol \& Blaes 1996). Unless the filling factor of magnetized regions in the disk photosphere is very low, our calculations suggest that optical radiation from AGN accretion disks will not be polarized. This of course begs the question of what is causing the observed optical polarization, and we would bet that it is dust and electron scattering in a stratified wind, as discussed by Kartje (1995). Polarization produced by the disk itself can only be observed by moving to shorter (ultraviolet) wavelengths.

\section{Absorption Opacity, Limb Darkening, and Polarization}

Ultraviolet spectropolarimetry has been carried out on a number of quasars with the Hubble Space Telescope and has revealed some remarkable surprises. Contrary to the predictions of Laor et al. (1990), the polarization is observed to steeply rise blueward of the Lyman limit in at least three objects (PG1222+228: Impey et al. 1995; PG1338+416, PG1630+377: Koratkar et al. 1995). In the case of PG1630 +377 the polarization rises to $\sim 20$ percent, well above the maximum value of a pure electron scattering atmosphere and therefore impossible to produce in a simple thin accretion disk model if the only thing that absorption opacity can do is decrease the polarization.

However, there are situations where this last statement is false. Polarization from a scattering atmosphere is related to the anisotropy of the emerging radiation field. In a pure electron scattering atmosphere, most of the radiation emerges vertically, i.e. the radiation field is limb-darkened. Some of the light emerging sideways was therefore scattered into this direction from an original vertical propagation direction, resulting in polarization (which is obviously par- 
allel to the disk plane). If the limb darkening could be enhanced, then it is more likely that the radiation emerging sideways was produced by scattering of originally vertically propagating radiation, and the polarization will increase. One way of accomplishing this is by having a steep thermal source function gradient combined with some modest absorption opacity. Then different viewing angles will see very different thermal source functions at unit optical depth along the line of sight, resulting in large limb-darkening and polarization. This effect of absorption opacity on polarization is not new - it has been explored in standard stellar atmospheres by Bochkarev, Karitskaya, \& Sakhibullin (1985).

Could this explain a steep rise in polarization blueward of the Lyman limit? To answer this question we have written a plane-parallel atmosphere code to calculate self-consistently both the accretion disk atmosphere structure and the emerging anisotropic, polarized radiation field (Blaes \& Agol 1996). All relativistic effects are neglected for now. Figure 1 compares the results of our calculations for both single temperature atmospheres and a multitemperature accretion disk with the data for PG1222+228. While none of the fits are quantitatively convincing, they certainly exhibit the qualitative behavior of the data. The polarization briefly drops blueward of the Lyman edge due to the large increase in absorption opacity but then rises steeply towards shorter wavelengths to values well above the Chandrasekhar value, to which all curves approach in the limit of zero wavelength where the absorption opacity vanishes. These models have effective temperatures such that the ultraviolet is in the Wien region of the Planck function. Any temperature gradient therefore gives rise to an exponentially steep source function gradient, at least within LTE. (Non-LTE effects do not appear to make much difference in the polarization, although they do change the total flux spectrum.) The polarization should still be parallel to the disk plane in these simple models, a prediction which has not yet been tested because of the lack of any observed radio axes in these sources.

\section{Conclusions}

Spectropolarimetry around the Lyman edge may be revealing the polarization signature of AGN accretion disks for the first time. While we have achieved a reasonable qualitative fit to $\mathrm{PG} 1222+228$, we have not yet been successful in explaining the other two sources - particularly PG1630+377. With ad hoc steep source functions, it is possible to produce the large polarization rise observed in this source, and more theoretical work needs to be done to find a physical cause for such atmospheres. In addition, three effects that we have neglected so far must be included: the relativistic transfer function (which might broaden the Lyman edge both in total flux and in polarization), Comptonization by a hot corona (which might also broaden the Lyman edge, cf. Czerny \& Zbyszewska 1991), and the effects on the disk atmosphere of external illumination by e.g. a hot corona. Clearly this problem is intimately tied to the problem of the apparent lack of Lyman edges in quasar spectra.

Acknowledgments. OB is very grateful to the RCfTA/ANUATC International Visitor Program for supporting his stay in Australia and enabling him to attend this conference. This research was supported in part by NSF grant AST 95-29230. 


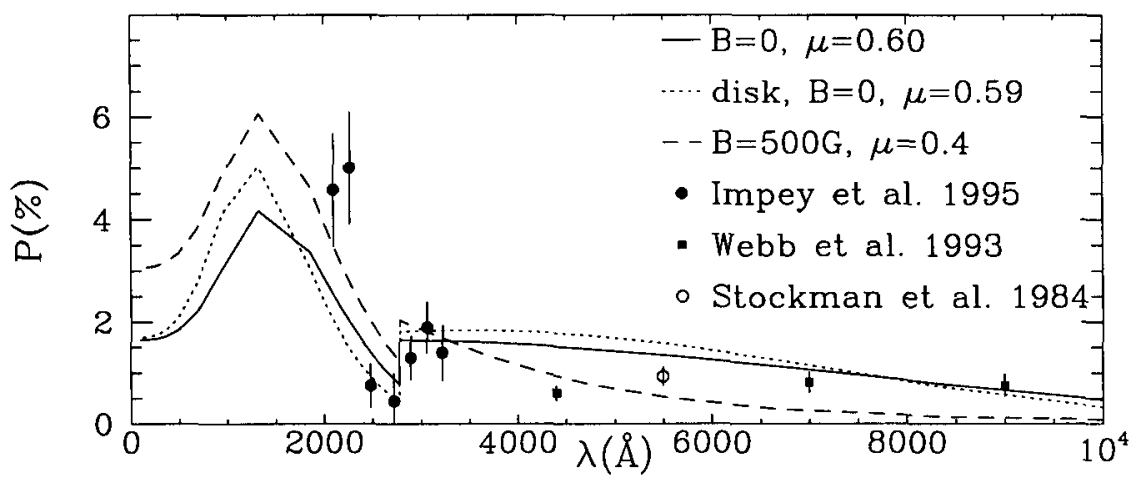

Figure 1. Polarization versus observed wavelength $(\mathrm{z}=2.047)$ of PG1222+228. The solid curve is a fit for a $T_{\text {eff }}=20,000 \mathrm{~K}$, $g=130 \mathrm{~cm} \mathrm{~s}^{-2}$ atmosphere model. The dashed curve shows a fit with the same model, but including Faraday rotation effects. Finally, a fit for a multitemperature disk model is also shown. Here $\mu$ is the cosine of the viewing angle with respect to the vertical or disk axis. (From Blaes \& Agol 1996.)

\section{References}

Agol, E., \& Blaes, O. 1996, MNRAS, in press

Antonucci, R. 1992, in Testing the AGN Paradigm, S. S. Holt, S. G. Neff, \& C. M. Urry, New York: AIP, 486

Barvainis, R. 1993, ApJ, 412, 513

Blaes, O., \& Agol, E. 1996, ApJ, 469, L41

Blandford, R. D. 1990, in Active Galactic Nuclei, T. J.-L. Courvoisier \& M. Mayor, Berlin: Springer-Verlag, 219

Bochkarev, N., Karitskaya, E. A., \& Sakhibullin, N. A. 1985, Ap\&SS, 108, 15

Chandrasekhar, S. 1960, Radiative Transfer, New York: Dover

Czerny, B., \& Zbyszewska, M. 1991, MNRAS, 249, 634

Gnedin, Yu. N., \& Silant'ev, N. A. 1977, Soviet Ast. Lett., 3, 136

Gnedin, Yu. N., \& Silant'ev, N. A. 1978, Soviet Ast., 22, 325

Impey, C. D., Malkan, M. A., Webb, W. \& Petry, C. E. 1995, ApJ, 440, 80

Kartje, J. F. 1995, ApJ, 452, 565

Koratkar, A., Antonucci, R. R. J., Goodrich, R. W., Bushouse, H., \& Kinney, A. L. 1995, ApJ, 450, 501

Laor, A., Netzer, H., \& Piran, T. 1990, MNRAS, 242, 560

Silant'ev, N. A. 1979, Soviet Ast., 23, 21

Stockman, H. S., Moore, R. L., \& Angel, J. R. P. 1984, ApJ, 279, 485

Webb, W., Malkan, M., Schmidt, G., \& Impey, C. 1993, ApJ, 419, 494 


\section{Discussion}

N. Arav: The Koratkar result does not show a drop in polarisation at the Lyman edge while your models show a drop. How can this be explained?

O. Blaes: This is an important point. In all our models the polarisation does indeed drop at the Lyman limit prior to rising steeply towards shorter wavelengths. This may be a problem in using our model to explain PG $1630+377$ although, as Koratkar points out, the signal to noise of the data needs to be improved to check this.

M. Livio: Can you please explain why in your model you don't see the reduction in polarisation due to relativistic effects, which was obtained by Laor et al.?

$O$. Blaes: We still do not include relativistic effects.

$R$. Wehrse: Since your models are $1 \mathrm{D}$, have you estimated the effects resulting from the finite disk extension in the other dimensions?

$O$. Blaes: We have not. My guess would be that breaking plane-parallel symmetry by disk flaring, for example, would reduce the polarisation. The magnitude of the effect would depend on the radial distribution of the emissivity. 